\title{
The Large Lecture Course Redesign Project: Pedagogical Goals and Assessment Results
}

Norman D. Aitken, (Email: aitken@provost.umass.edu), University of Massachusetts Amherst

\begin{abstract}
An analysis and assessment of the Course Redesign Project, which used technology to improve student learning and course satisfaction in large lecture courses at the University of Massachusetts Amherst. Six disciplinary-diverse departments participated in the project. Technology was selected for the purpose of introducing active learning into lecture halls and providing frequent feedback to students on their individual learning progress. The assessment methodology compares traditionally taught sections with redesigned sections, holding constant (where possible) such potential confounding factors as student academic ability, professor, textbook, day and time of class and the number, type and difficulty of exams and other graded assignments. The assessment of the project produced strong and significant statistical results that indicate that students across the broad spectrum of redesigned courses learned more and achieved higher grades than students in traditional sections. This occurred despite the fact that students in traditional sections had either the same or higher high school-grade point averages and SAT scores compared to students in the redesigned sections. The project included 12 traditional course sections with a total enrollment of 2,456 and 13 redesigned courses sections with a total enrollment of 3,101. The project was supported by a grant from the Davis Educational Foundation.
\end{abstract}

\section{Introduction}

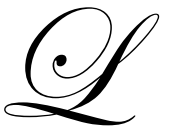

ike most large institutions of public higher education, The University of Massachusetts Amherst teaches many introductory courses in a large lecture format, where hundreds of students are taught by single professors, with varying degrees of Teaching Assistant support. While this traditional approach has made efficient use of faculty, it has not provided the best learning experience for students. Recent studies of student learning confirm that didactic presentation (where faculty lecture and students listen passively) provide an inferior learning experience for students (e.g., see Cuseo, Joe; "The Empirical Case Against Large Class Size: Adverse effects on the teaching, learning and retention of first-year students"). While lecturing may continue to be an important component to teaching, these studies recommend that courses be redesigned to include at least the following pedagogical components: 1) active learning experiences; 2) frequent assessment of learning; 3) meaningful and frequent feedback to students on their learning process and 4) relating course content to the personal experience of students. Prior to the advent of the information technology (IT) revolution, these pedagogical changes were difficult and expensive to implement because they required additional teaching personnel to handle the additional work involved; thus raising the total cost of instruction. The primary goal of the Course Redesign Project was to improve the learning experience of students in large lecture courses, by using modern technology to incorporate the above specified pedagogical components into the course. This was to be achieved without raising the cost of instruction, except for new instructional technology funded by the University in the form of personal response systems installed in 
lecture halls. A second major goal of the project was to assess the impact of the redesign on student learning and student satisfaction. The project was supported by a grant from the Davis Educational Foundation.

This paper is divided into three major sections: 1) Project Implementation, which describes the courses and faculty involved in the project and the pedagogical approach and process by which the courses were redesigned., 2) Assessment, which covers the assessment process and statistical results and 3) Summary and Conclusions:

\section{Project Implementation}

\section{Course Selection}

The Project began in the spring semester 2002 with the selection of courses, faculty and departments who would participate in the project. The overall design of the project was to select six large lecture courses from different departments which would cover a wide variety of disciplines. Proposals for course redesign submitted by departments were reviewed by a committee comprised of faculty and professional personnel known for their expertise in undergraduate teaching. Criteria for selection included commitment to the goals of the project, teaching reputation of faculty, quality of proposal, and size of course enrollment.

The courses and corresponding academic departments selected for the project are as follows:

\section{Course Title}

Intro to the Visual Arts

Exploring the Universe

Intro to Microeconomics

Corporation Finance

Intro to Psychology

Intro to Statistics
Course Number

ArtHist 115

Astro 100

Econ 103

FOMGT 301

Psych 100
Department

Art History

Astronomy

Economics

Finance and Operations Management

Psychology

ResEc 211/212

More detailed information is provided in Table 1 in the Appendix, where a number identifier is used for individual faculty to provide anonymity.

It was decided that each participating department would teach a traditional section(s) of their course in order to obtain baseline data against which the redesign sections could be compared. The Economics and Finance and Operations Management Departments each had one participating faculty member, who taught both a traditional and redesign section of their designated course. The other four departments had multiple instructors and in each department at least one faculty member taught both a traditional and redesigned section to provide opportunities for statistical comparisons of different teaching models using the same professor. In order to provide more extensive background data, the Resource Economics Department also used course data they had captured for other purposes in the fall 2001 and spring 2002 semesters.

After the participating departments and courses were selected, the remainder of the spring 2002 semester was devoted to weekly meetings to plan the redesign for each course. By meeting as a group, the participating faculty were also able to focus on issues that impacted the project as a whole (i.e. all redesigned courses) as well as learn new teaching pedagogy and technologies to use in their individual 
classes. Through these weekly meetings a consensus was developed among all participating faculty to include the following components in all redesigned courses: 1) the use of the Personal Response System (PRS) and 2) Online computer quizzes to be completed prior to class, ultimately to be delivered to students through the University’s Online Web-based Learning (OWL) System.

\section{The Personal Response System (PRS)}

PRS involves the use of wireless transmission from a personal transmitter for each student to receivers installed in the lecture hall. The receivers then send the individual student answers to the laptop computer of the professor. Because the PRS transmitters have a microchip with a unique identifying number, the professor's computer is able to record the response of each student individually. The computer's software then aggregates the responses for all students and can project them in the lecture hall when the professor so chooses. Agreement by all project faculty to use the system enabled the University to begin installing receivers in lecture halls during the summer of 2002, so that the system would be ready for use in the redesigned courses in the fall 2002 semester. The University would continue installing more PRS systems in lecture halls in subsequent semesters. As of fall 2003, PRS systems were available in thirteen classrooms ranging in seating capacity from 150 to 500, with a total capacity of 3,200. The availability of PRS-ready classrooms provides considerable flexibility for faculty on campus to use PRS in their classes without being constrained by class time or location.

\section{Online Quizzes}

Prior to the start of the project several faculty had been using online quizzes in their courses. These were being delivered through commercial software (WEBCT or Blackboard). As part of the project the faculty participants compared these systems to the Online Web-based Learning (OWL) system that was provided on campus through the Center for Computer Based Instructional Technology (CCBIT). Eventually all faculty participating in the project adopted OWL as their quizzing tool. While the type of quiz questions presented through OWL varied by discipline, all faculty through independent decisionmaking arrived at a fairly standard approach to using the OWL online quizzes. Eventually the quizzes were commonly referred to as "Pre-Lec" quizzes. The quiz questions were on the assigned reading for each lecture and were due prior to the lecture. Since these quizzes could be taken from student dorm rooms, the Pre-Lec quizzes were not viewed by faculty as a valid testing tool, but rather as an incentive to have students read the material prior to coming to the lecture (not a common practice for many college students). Some faculty allowed or even encouraged students to use their textbook in answering the PreLec questions because this would familiarize students with the major concepts that were going to be included in the lecture. An informal conversational poll among faculty indicates that using the Pre-Lec quizzes resulted in students being better prepared for class and allowed the faculty member to cover the lecture content more rapidly and effectively. PRS quizzes during class then allowed the faculty member to test and measure the extent to which students actually understood the material being presented in the lecture. Faculty view the Pre-Lec quizzes and the PRS quizzes as an effective combination of learning tools which encourage students to be prepared for class and then tests student knowledge of the material

during the class. Essentially, the faculty see the Pre-Lec OWL quizzes as a learning tool rather than a testing device. Exam testing still occurs in the lecture halls, where students are closely monitored, using either written or multiple-choice exams. 


\section{Assessment}

\section{Assessment Plan}

A well designed assessment plan was of critical importance to both the University and the Davis Educational Foundation. The assessment plan included the following components: 1) A large number (sample) of students so that valid statistical tests could be conducted with a high probability that they would be able to effectively measure any changes resulting from the project. 2) An experimental design which would capture "before and after" data to further enhance the likelihood of valid measures and tests of the effectiveness of the project. 3) The collection of comprehensive data on student performance and ratings of courses taught in the traditional and redesigned formats. 4) The use of powerful statistical tools to test for the various outcomes of the project.

Based on the above goals, the assessment plan was designed to avoid introducing so-called "confounding factors" into the analysis. Of particular importance was the individual faculty member. If traditional courses were compared to redesigned courses with different faculty, it would be hard to conclude that the redesign itself was the factor that changed student learning outcomes (rather than the difference in faculty). On the other hand, faculty teach courses in departments according to departmental staffing needs and it was clear that it would not be possible to have all participating faculty teach both a traditional and a redesigned course. Fortunately, for each course, at least one faculty member agreed to teach both a traditional and redesigned section of the course. In one case (Psychology) the faculty member taught both the traditional and redesigned sections of the course in the same semester. In all other cases, the faculty member(s) taught the traditional course in one semester, followed by the redesigned course in a subsequent semester. Additional faculty in four of the departments taught either a redesigned or traditional section of the course. The reader should keep these distinctions in mind in reading the assessment results which follow. We will report the results separately for the course sections taught by the "same" faculty and the aggregate results for all traditional and redesigned sections, whether taught by the same faculty member or different faculty members. To briefly anticipate our findings, the results are very similar whether the traditional and redesigned sections were taught by the same faculty member or different faculty members.

Because we were studying large lecture courses and because we collected data on multiple sections, we were able to achieve the goal of large sample sizes. Adding up the individual enrollments from Table 1 shows that there were 2,456 students enrolled in the traditional sections and 3,101 in the redesigned sections for a total student sample of 5,557. These numbers are for enrollment, the actual sample size for each statistical test varied because not all students answered the surveys that were administered online. There was also some data missing from the student data base on particular student characteristics. These numbers were generally small, but the reader should be aware that the sample size does vary depending on the particular statistical test being reported. However, in all cases the sample size remains large. Two basic statistical techniques were used to analyze the data: 1) means and standard deviations for cardinal data and numerical totals, and 2) percentages for categorical information. The results for traditional sections versus redesigned sections were then compared using t-tests for the difference between means and Chi Square tests for the difference between proportions. Two-tailed tests were then used to determine whether the differences between traditional and redesigned courses were statistically significant. Multiple regression analysis was also used to test the hypothesis that the redesigned sections resulted in higher student-course grades. For all of these tests, the standard for considering the results statistically significant was a probability of .05 or less. In other words, the 
observed differences had to have a probability of 5 out of 100 (5\%) or less that they could have occurred by chance in order to be declared statistically significant.

\section{Assessment Results}

The combined data set for this project included 355 variables (the sum of student responses to particular survey questions and individual student characteristics obtained from the University Student Data Base). Clearly, a huge amount of data was amassed on students who enrolled and participated in the courses. It is not realistically possible to analyze every single component of this data. Selected for analysis, therefore, are those variables that are most important for assessing the overall impact of the project. Primary focus has been placed on student academic performance in the courses (final course grade) and student ratings of the faculty member's teaching characteristics and the various components of the course as seen through student ratings. Also of prime importance is how students viewed the use of the PRS system and the Online Quizzing component in the redesigned courses. Finally, we compared characteristics of students in the redesigned sections versus the traditional sections. If students in the redesigned sections have stronger academic traits than students in the traditional sections, this could explain better academic performance, independent of the effectiveness of the redesign.

The statistical results for the assessment are contained in Tables 2A through 7 in the Appendix. The extent to which students in the redesigned sections may have been better prepared for college work, as measured by SAT scores and high school grade point average, is addressed in Tables 2A and 2B. Table 2A compares students in traditional and redesigned sections for courses taught by the same professor and Table 2B makes the same comparison for course taught by all faculty members. Table 2A shows that the high school grade point average of students in traditional and redesigned sections were virtually the same (3.28 vs. 3.29), resulting in a low and insignificant t-value. On the other hand, the verbal, math and combined SAT scores are significantly higher for students in the traditional sections than in the redesign sections. The Project course grade average, however, is significantly higher in the redesigned course sections. Looking at Table 2B for all course sections we find very similar results and no major inconsistencies between the two tables. The results here are very strong. They indicate that the students in the traditional sections had academic characteristics that were as strong as, if not stronger, than the students in the redesigned sections. However, students in the redesigned sections actually performed better, as indicated by the final grade in the course. Where both traditional and redesigned sections were taught by the same faculty member, the textbook, the reading assignments and the grading procedures would have been the same. Hence, the results strongly support the conclusion that the redesigned section of the courses taught by the same faculty members resulted in improved student learning and performance. The results for all faculty suggest that this phenomenon of improved student performance occurred for the project as a whole, including traditional and redesigned courses taught by different faculty members.

The results for the individual courses are consistent with the aggregate findings. Students in the redesign sections for this project did not have stronger academic profiles than the students in the traditional sections and, therefore, this could not have explained the higher grade performance in the redesigned courses. To conserve space, the individual course results are not reported in this paper, but are available from the author upon request.

Table 3 shows the results from a different statistical tool, multiple regression analysis, which tests for direct statistical relationships between course grade as the dependent variable and four independent variables: high school grade point average, math SAT scores, verbal SAT scores, and whether the course 
was traditional or redesigned. The latter is entered as a "dummy" variable with a value of one for students in redesigned courses and a value of zero for students in traditional courses. The regression model simultaneously captures the effects of high school GPA and SAT scores, and hence, any effect shown by the traditional/redesign variable is a powerful test of its effect on course grade. As can be seen in Tables 3A and 3B, all independent variables for both same faculty and all faculty members are statistically significant. The results show a strong grade effect for students being in redesigned courses. In other words, students in redesigned courses are likely to earn a higher course grade regardless of their high school academic characteristics.

Tables 4A and 4B show aggregate student ratings of several professor characteristics, as reported by students in the end of semester surveys. In interpreting these tables it is important to recognize that smaller values indicate higher student ratings of the particular item. Both tables show statistically significant higher ratings on the following items: Professor used class time well; Overall rating of professor's teaching; Professor inspired excitement; and Professor gave regular feedback.

Tables $5 \mathrm{~A}$ and $5 \mathrm{~B}$ contain aggregate results for student ratings of three aspects of the course itself: 1) workload, 2) how much learned in the course, and 3) overall course rating. The traditionalredesigned comparison was statistically significant for only one rating, the workload for the course. The students in the redesigned sections rated workload for their course as being significantly heavier than the students in the traditional sections taught by the same faculty member. In the results for all faculty members, students in the redesigned sections also rated their workload as heavier than students in the traditional sections. Although this result was not statistically significant, it had a relatively high t-value (1.31). There were no statistically significant differences in the aggregate results for the last two questions in these tables (how much learned; overall course rating.

\section{Student Ratings of the Personal Response System (PRS):}

Use of the Personal Response System to record individual student answers in an interactive setting within the lecture hall was one of the two major forms of technology used in the Large Lecture Course Redesign Project. PRS was used in twelve of the thirteen redesigned course sections (the thirteenth course, ResEc 211 adopted PRS in fall 2003). The online student survey attempted to assess the student experience with PRS by including six questions on the topic. Students were asked to use a four-category scale to indicate their rating of PRS in the lecture hall. The answers ranged from "strongly agree," to "strongly disagree" on a four-point scale, and the specific values are listed at the bottom of Table 6. The smaller the value of the student answer, the more they tended to agree with the statement on PRS. The statements themselves are also listed in Table 6. Note that the first four statements are positive, while the last two are negative. For each statement, the table reports the percentage of students who agreed ("strongly agree" or "agree”) or disagreed (“disagree” or "strongly disagree”) with each statement. The table also shows the mean rating for all respondents for each statement. Finally, the table shows that approximately 2,370 students answered each of these survey questions. ${ }^{1}$

Only a slight majority (54\% vs. 46\%) of students agreed that they were better prepared for class because they would need to answer PRS questions in the lecture. The average student rating for this statement was 2.46, falling almost midway between agreeing and disagreeing. This distribution of answers does not seem unreasonable because online quizzes, which students completed before class, were targeted as the required task to ensure that students were prepared for the lecture. On the other hand, the PRS questions in the lecture hall were usually directly related to what the faculty member was teaching in

1 The number of respondents varies slightly around 2,370, since not all students answered each question. 
that class. The responses to Statement 2 seems to confirm that interpretation, because $75.71 \%$ agreed that they "learned more in class" because PRS questions made them apply what the professor taught during the class. Only $24.29 \%$ disagreed with this statement. The average response was 2.06 indicating agreement with the statement. The results for Statement 3 show that $66.61 \%$ of the students agreed that "using PRS was fun and made the class more enjoyable." The mean response for this item was 2.20, which falls into the agree range on the rating scale. A significant majority (66.93\%) also agreed with the statement that "answering PRS questions encouraged me to talk to other students." The mean rating for this statement was 2.19, also falling into the agree range on the rating scale. The two negative statements (5 and 6) show a majority of students disagreeing. Fifty-nine percent of the respondents disagreed with the statement that PRS did not significantly improve their "learning in this class." The mean rating was 2.63, falling into the slightly disagree range. The rather blunt statement, "Using PRS was a waste of time," showed that $73.75 \%$ of the students disagreed with it. This statement had a mean rating of 3.00, also indicating significant disagreement.

The strength of these results provides clear and strong evidence that the introduction of the Personal Response System in the lecture halls encouraged students to talk to one another, made the class more enjoyable, and helped students learn more in class. Even though students had to buy their PRS transmitters for \$36, 74\% did not believe using PRS was a waste or time.

\section{Student Ratings of Online Quizzes}

During the fall 2002 semester a number of faculty were still teaching traditional courses, while others had begun teaching redesigned courses, and a number of these faculty were still using online quizzes delivered through WebCT or Blackboard. Hence when the student survey was constructed, questions were worded in a generic way to ask about student experience with online quizzes. " Rate the usefulness of the online quizzes taken over the internet (e.g., OWL, WebCT, Blackboard or other) in helping you learn. Tables 7 report the results for this question, both as mean ratings and as the percentage breakdown for each answer. Remembering that small numbers here are the most favorable ratings $(1=$ "extremely useful"), students rated the usefulness of online quizzes over the internet at 2.89, which indicates that they found online quizzes usually useful.

\section{Summary and Conclusions}

In general, faculty who have participated in the Course Redesign Project are satisfied with the courses that they have redesigned and are continuing to use the redesigned components in their current teaching. This is especially true of the PRS system and online quizzes delivered through OWL. Faculty also believe that further improvements in teaching and learning can occur as a result of this project as they create better questions and learning modules for these systems, respectively. The use of the PRS system developed by the Course Redesign Project has now spread well beyond the project itself, with twelve additional faculty using it in their large lecture courses enrolling 3,300 students in addition to those in the Course Redesign Project Fall 2003 courses. We anticipate that PRS usage will continue, and that even more faculty will adopt the PRS system or equivalent technique in future semesters. Students in the redesigned courses and other similar courses on campus now seem to be satisfied with the use of the PRS transmitter in the large lecture classroom. Students in the redesigned sections also rated their workload as heavier than students in traditional sections, which is consistent with our finding that they actually learned more in these classes. Finally, and perhaps most importantly, the assessment of the project produced strong and significant statistical results that indicated that students across the broad spectrum of 
redesigned courses learned more and achieved higher grades than students in the traditional sections. This occurred despite the fact that students in traditional sections had either the same or higher high school grade point averages and SAT scores than students in the redesigned sections.

In conclusion, the Large Lecture Course Redesign Project has made positive and lasting changes on the teaching of students in large lecture courses. A critical mass of faculty has now changed the way they teach and students now see lecture halls as a place for active learning and direct feedback on their learning progress. Students believe that they work harder than before, but see the effect of their work in the form of higher grades.

\section{Appendix}

\section{Statistical Tables 1-7}

